\title{
MAGNETO-OPTICAL MEASUREMENTS USING AN OPTICAL FIBER AND HYBRID MAGNET SYSTEM
}

\author{
G. KIDO and Y. NAKAGAWA \\ Institute for Materials Research, Tohoku University, Sendai 980, Japan
}

\section{ABSTRACT}

Magneto-absorption spectra have been measured in the steady magnetic fields up to $27 \mathrm{~T}$ by means of a hybrid magnet at Tohoku University. For the distant light transmission, bundle optical fibers were employed for the rapid assembly of the light pass within a restricted machine time. Both the Faraday and voigt configurations were realized in a tail of the cryostat with the longitudinal magnetic field. The monochromator and optical filters were fully remote controlled from outside of the magnet room. Pressure dependence of the luminescence spectra was also measured in the magnetic field up to $14 \mathrm{~T}$ using a water cooled resistive magnet.

\section{INTRODUCTION}

Magnetic field effects on absorption spectra have been actively investigated in various materials containing magnetic ions. The field dependence of absorption lines provides us much information concerning localized excitations, excitons, magnon sidebands etc. Intense magnetic fields have a possibility to bring about a drastic change of spectra with respect to the spin phase transition. Pioneer in this field attempted to use pulsed magnetic field combined with a xenon flash and photography $[1,2]$. Recently, superconducting magnets have been used conventionally up to $15 \mathrm{~T}$, which made possible to measure high resolution spectra [3]. However, much higher field is left to a resistive magnet with huge electric power consumption at present.

A hybrid magnet was devised to generate practically the highest steady magnetic field using the resistive magnet together with a superconducting magnet. In Japan, three hybrid magnets have been constructed in Tohoku University [4]. The world highest record of 31.1 T was achieved in 1986 by the use of strongest magnet ( $\mathrm{HM}-1 \mathrm{a})$ among them [5].

Previous to the success, we have planned to make a system to measure the magneto-absorption spectra [6]. Since the hybrid magnet consists of a large and strong superconducting magnet, an effect of leakage flux extends several meters far from the magnet to a detector, monochromator and laser. On the other hand, immediate setting of the light pass is essential for the measurements within a restricted machine time. We developed a magneto-optical spectrometer using an optical fiber system for the distant light transmission, and successfully measured absorption spectra in the steady magnetic field up to $27 \mathrm{~T}$.

\section{HYBRID MAGNET}

The cross section of the hybrid magnet $\mathrm{HM}-1$ is shown in Fig. 1. A superconducting magnet surrounds a resistive magnet coaxially. The inside of the superconducting magnet is at ambient temperature, whose diameter

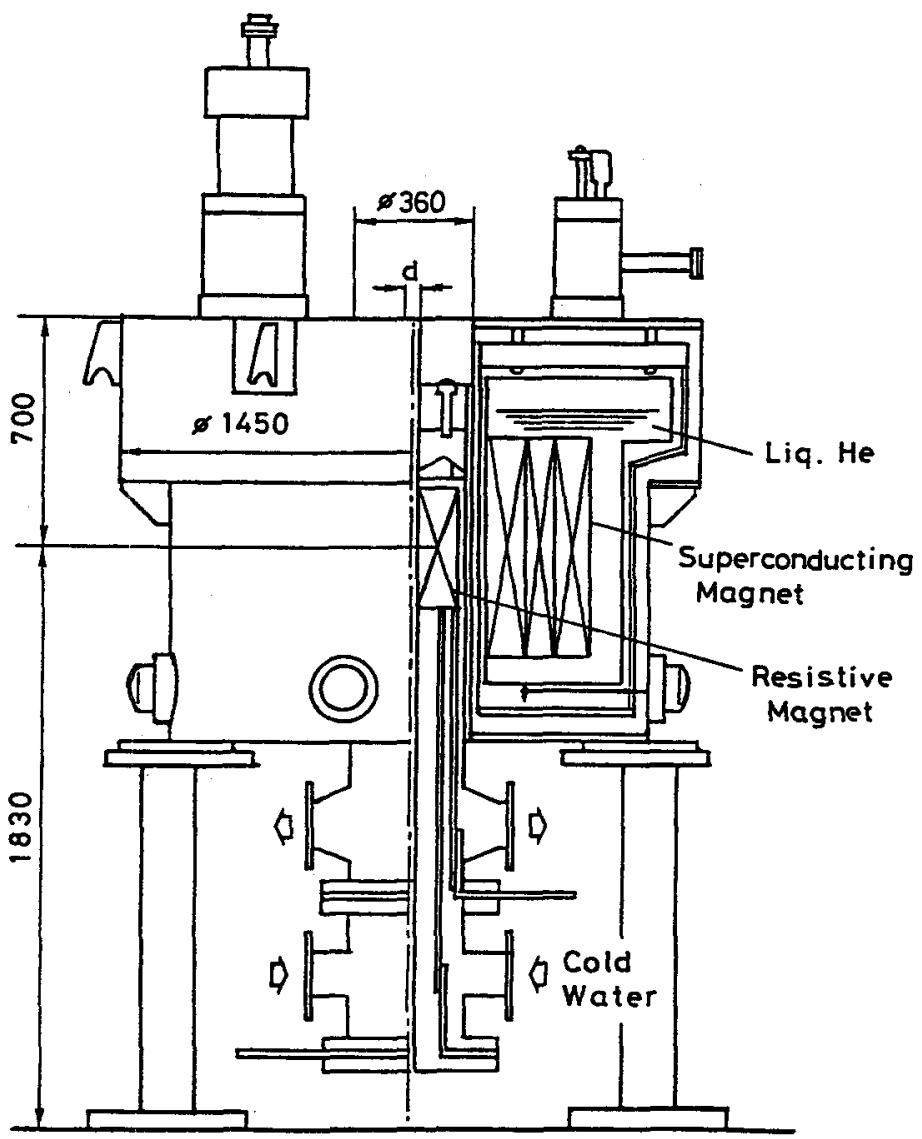

Fig. 1. Cross section of the hybrid magnet HM-1. 
is as large as $360 \mathrm{~mm}$. The superconducting magnet is graded in three layers. By the use of multifilamentally $\mathrm{Nb}_{3} \mathrm{Sn}$ wires in the inner layers of superconducting magnet, the magnetic field up to 12 $T$ can be generated. Two kinds of polyhelix coil with different inner diameter are employed for the resistive magnet. The resistive coil is excited by dc-power supply of 8 megawatts and cooled by flowing cold water of 100 liters per second.

In the hybrid mode, the resistive magnet is excited after the excitation of the superconducting magnet. The magnetic field up to $31.1 \mathrm{~T}$ can be generated with $32 \mathrm{~mm}$ clear bore (HM-1a). The maximum field reduced to $28.1 \mathrm{~T}$ when using resistive magnet of $52 \mathrm{~mm}$ bore (HM-1b). The field strength was determined by the absolute measurement using an induction method [7]. The optical measurements were carried out using HM-1b hybrid magnet. Smaller hybrid magnet HM-2, which is able to generate up to $23 \mathrm{~T}$, and a resistive magnet up to $15 \mathrm{~T}$ were also employed for the lower field experiments.

\section{CONFIGURATION}

An optical system of the magneto-absorption spectrometer by means of a hybrid magnet is shown in Fig. 2. Flexible bundle optical fibers lead light from monochromator to a cryostat and from the cryostat to a detector. In the cryostat, single optical fibers with a large core of $1 \mathrm{~mm}$ in diameter are employed for the light transmission. One bundle fiber consists of a hundred thin quartz fibers of $80 \mu \mathrm{m}$ in core diameter and $125 \mu \mathrm{m}$ in clad diameter. The thin fibers are aligned straight at the monochranator side in order to use the monochromatic light effectively through the exit slit. A specially made mirror box is attached to the exit of the monochromator. The bundle fibers and the single fibers are made by The Furukawa Electric Co. Ltd. and Mitsubishi Electric Wire Co. Ltd., respectively.

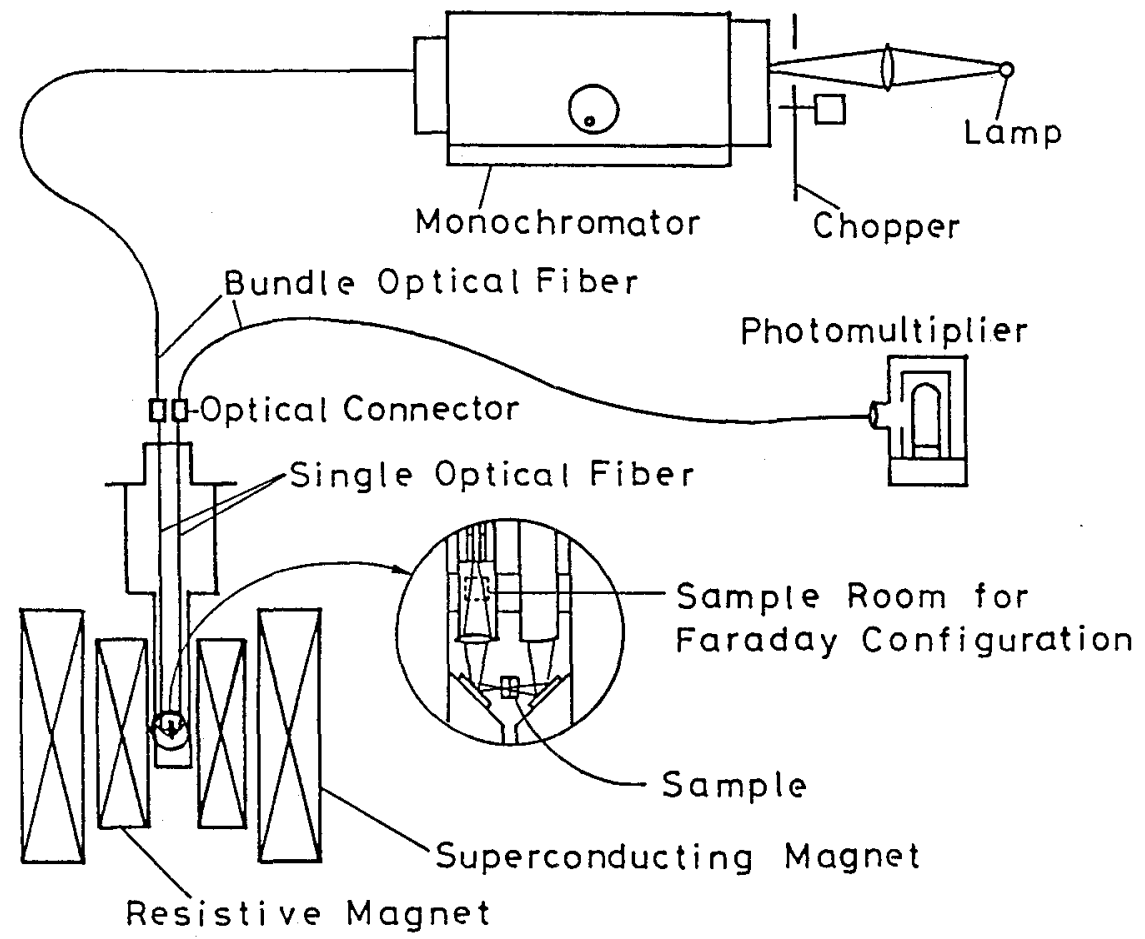

Fig. 2. Absorption spectrometer using a optical fiber system combined with a hybrid magnet.

A tail of the cryostat is inserted in the hybrid magnet. The sample is placed near the bottom in the tail and is immersed in liquid helium. The temperature of the sample is varied down to $1.3 \mathrm{~K}$ by evaporating the liquid. The transmitted light through the optical fiber is focused down to the sample by the convex lens. The light is hit by two plane mirrors and re-focused on the end of another optical fiber. The Voigt configuration is realized by placing the sample between the mirrors. When the Faraday configuration is required, the sample is mounted near the end of the optical fiber. The light can be polarized by inserting a polaroid sheet in the light pass just close to the sample.

Since the absorption in the optical fiber is not negligible in wide wavelength region, we restricted the total length of fiber within $20 \mathrm{~m}$. Consequently, the monochromator had to be placed in the magnet room. Figure 3 shows an actual configuration of the magneto-absorption spectrometer. The monochromator is placed six meters away from the hybrid magnet HM-1. The leakage flux at that point was less than $0.01 \mathrm{~T}$. No troubles arose for the scanning of the wavelength. The JASCO CT-50 monochromator is remotely controlled by a computer from outside of the magnet room. The field strength is adjusted by the currents in superconducting and resistive magnets, respectively. The output signals from the detector and the wavelength are memorized in the computer and are converted into the absorption or luminescence spectra. 


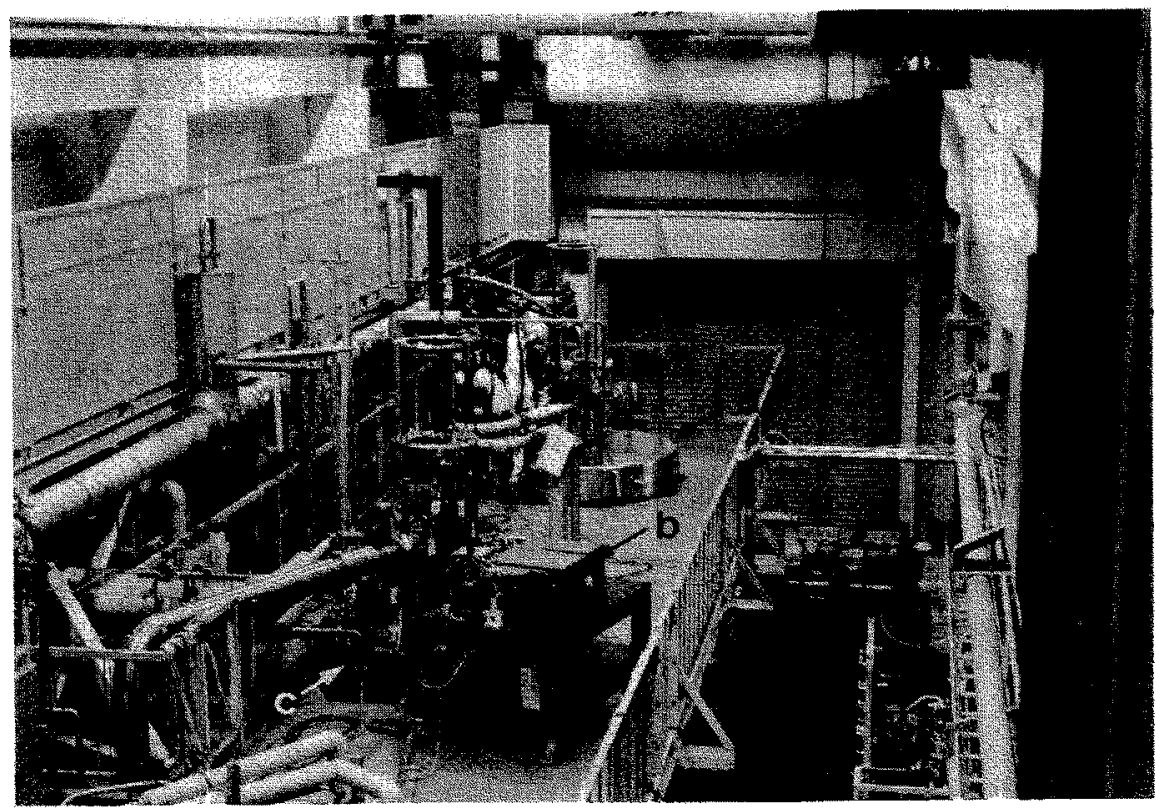

Fig. 3. Photograph of the hybrid magnet room. Arrows indicate a) HM-1 magnet, b) monochromator and c) HM-2 nagnet.

Luminescence spectra were also measured in high magnetic fields. An argon ion laser was used for the excitation of a sample. The laser beam was led by a single fiber ( $80 \mu \mathrm{m}$ in diameter) to the sample. A compound glass fiber (Showa Electric Wire \& Cable Co. Ltd.) was employed for the laser beam transmission, since it does not accompany large luminescences by itself. The emission from the sample was corrected by a lens and transmitted to the monochromator by the similar light system mentioned in the absorption measurement. The pressure dependence of the spectra can be obtained more easily in the luminescence rather than the absorption measurements. We employed a resistive magnet with large bore of $82 \mathrm{~mm}$ where our diamond anvil can be mounted. The Iuminescence spectra were measured up to $14 \mathrm{~T}$. Details

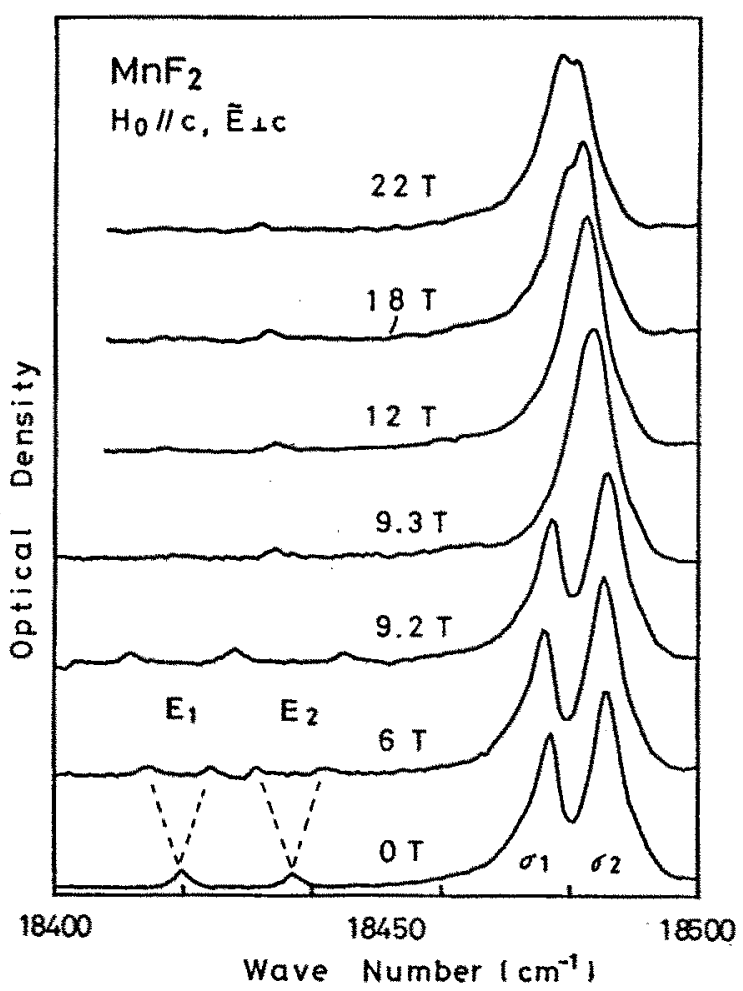

Fig. 4. Absorption of exciton and magnon sideband of $\mathrm{MnF}_{2}$ at $4.2 \mathrm{~K}$ in the polarization. have been described in a separate paper [8].

\section{EXAMPLES OF MAGNETO-ABSORPTION SPECTRA}

The absorption spectra corresponding to the transition $6_{\mathrm{A}_{1 \mathrm{~g}}}-{ }_{\mathrm{T}_{1 \mathrm{~g}}}$ of $\mathrm{Mn}^{2+}$ in the $\mathrm{MnF}_{2}$ crystal are exhibited in Fig. 4, where the magnetic field is apolied parallel to the caxis. $\mathrm{MnF}_{2}$ is the antiferromagnet $\left(\mathrm{T}_{\mathrm{N}}=67 \mathrm{~K}\right)$ having the rutile structure. The magnetic moments are parallel to the c-axis. Four absorption lines are observed at zero magnetic field in the $\sigma$ polarization: the propagation direction $(\bar{k})$ and the electric vector $(\bar{E})$ of light are both perpendicular to the c-axis. The measurements are carried out in the voigt configuration. Two strong absorptions denoted to $\sigma_{1}$ and $\sigma_{2}$ are the magnon sidebands of $E_{1}$ and $\mathrm{E}_{2}$ exciton lines, respectively [9].

The magnon sidebands renain at the same energy up to $9.2 \mathrm{~T}$ just below the spin flop field of $9.24 \mathrm{~T}$ where the spins rotate to be perpendicular to the c-axis. These lines discontinuously approach each other at $9.3 \mathrm{~T}$ and shift to lower energy above the transition. The sublattice splitting is displayed in the exciton lines up to the transition field. Above the transition the exciton lines show no splitting since the magnetic field energy is identical for the $\mathrm{Mn}^{2+}$ spins. It was found for the first time that $\mathrm{E}_{2}$ line considerably shifts in proportional to the square of the magnetic field, while $\mathrm{E}_{1}$ remains at the same position above the transition. 
In the $\pi$ polarization $(\overline{\mathrm{E}} / / \mathrm{c}, \widetilde{\mathrm{k}} 1 \mathrm{c})$, the magnon sideband $\pi_{1}$ appears, which is also related to the $E_{1}$ exciton. This line is almost insensitive to the magnetic field up to $22 \mathrm{~T}$ as shown in Fig. 5. Details of the energy shift and splitting were described in the previous report [6].

Figure 6 shows another example of magneto-absorption spectra in a onedimensional antiferromagnet $\mathrm{CsCoCl}_{3} \cdot 2 \mathrm{H}_{2} \mathrm{O}$ crystal. Linear chains of $\mathrm{Co}^{2+}$ ion extend in the a-direction by sharing $\mathrm{Cl}^{-}$ion, so that strong superexchange interactions act between the neighboring $\mathrm{Co}^{2+}$ ions. The magnetic fields are applied along the c-axis which is almost parallel to the magnetic moments. The magnon sideband denoted at $\mathrm{B}_{1}$ line shifts lower energy side up to $14 \mathrm{~T}$. The absorption intensity of $\mathrm{B}_{1}$ line much decreased at $27 \mathrm{~T}$ where all the magnetic moments are aligned to the external magnetic field. The absorption spectra with respect to the magnetization are analyzed in detail in the separate paper [10].

\section{CONCLUSION}

An absorption spectrometer was developed using an optical fiber system for the distant light transmission, and was combined with a hybrid magnet system built at Tohoku University. The absorption spectra were successfully measured in various substances in the steady magnetic fields up to $27 \mathrm{~T}$. Luminescence spectra under the high pressure were also measured in the field up to $14 \mathrm{~T}$ using a resistive magnet.

\section{ACKNOWLEDGMENTS}

The authors wish to thank Professor $\mathrm{K}$. Aoyagi for the collaboration in the works on $\mathrm{MnF}_{2}$. They also express their thanks to Professor I. Tsujikawa and Dr. N. Kojjma for the collaboration on $\mathrm{CsCoCl}_{3} \cdot 2 \mathrm{H}_{2} \mathrm{O}$. Thanks are also due to Professors $\mathrm{Y}$. Nishina and $\mathrm{N}$. Kuroda for cooperation on study of magnetoluminescence. Authors are grateful to R. Tanaka, M. Takeda for their contribution in the experiments. They are indebted to the members of Cryogenic Center and High Field Laboratory for Superconducting Materials of Tohoku University. This work was partly supported by the Grant-in-Aid for Scientific Research from the Ministry of Education, Science and Culture of Japan.

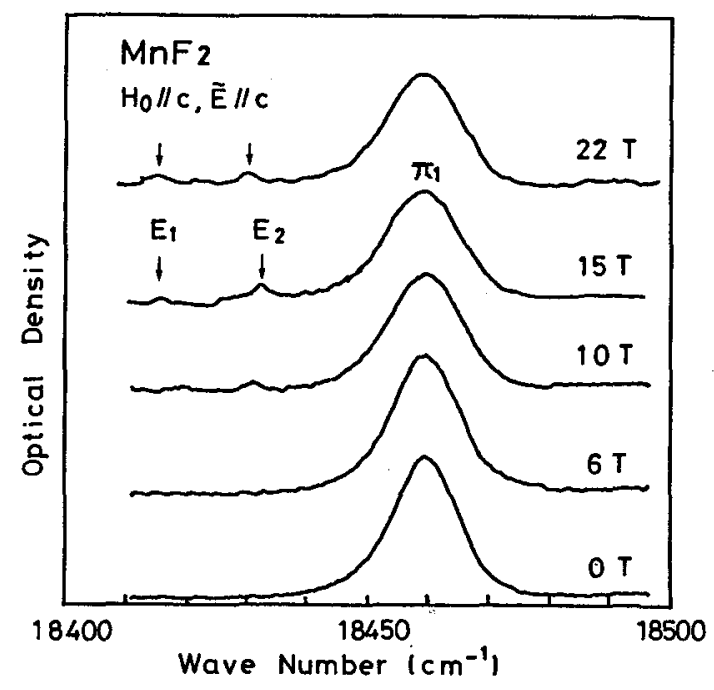

Fig. 5. Absorption of $\mathrm{MnF}_{2}$ in the $\pi$ polarization.

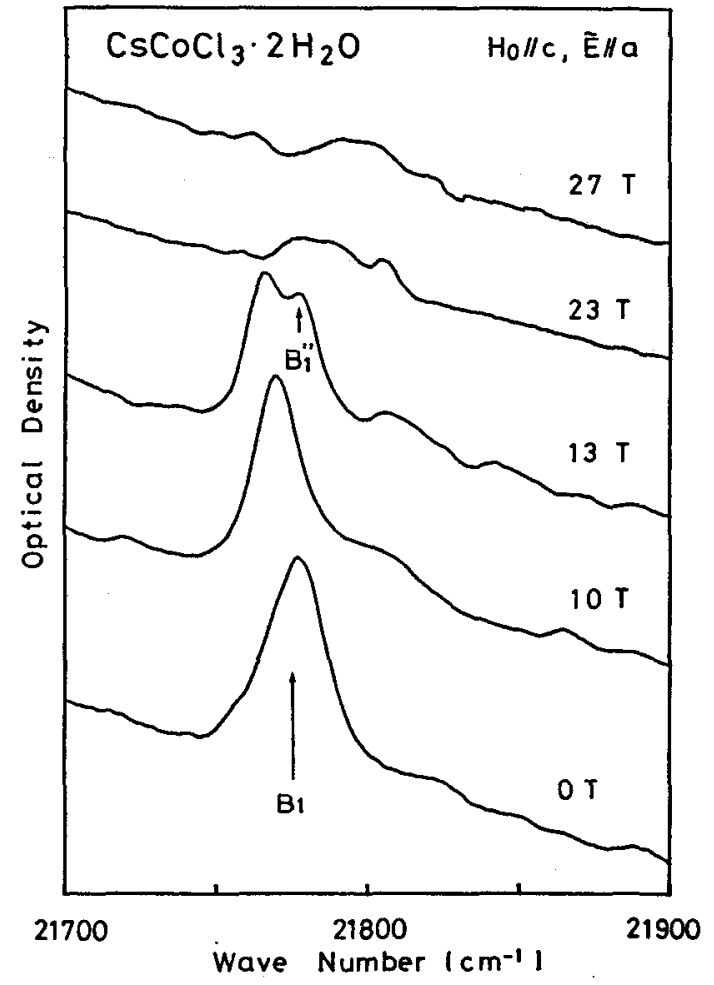

Fig. 6. Behavior of magnon sideband in $\mathrm{CsCOCl}_{3} \cdot 2 \mathrm{H}_{2} \mathrm{O}$.

\section{REFERENCES}

[1] K. Aoyagi, A. Misu, S. Sugano, J. Phys. Soc. Jpn. 18 (1963)1448.

[2] P. G. Russel, D. S. McClure and J. W. Stout, Phys. Rev. Lett. 16 (1966) 176.

[3] J. Nakahara, A. Fujii, J. Phys. Soc. Jpn. 48 (1980) 1184.

[4] Y. Nakagawa, K. Noto, A. Hoshi, S. Miura, K. Watanabe and G. Kido, Proc. Inter. Conf. Magnet Technology (Zurich, 1985) p. 458.

[5] Y. Muto, Y. Nakagawa, K. Noto, S. Miura, A. Hoshi, K. watanabe, G. Kido, H. Ishikawa, T. Fujioka, Y. Sato, O. Osaki and H.Takano, Sci. Rep. RITU, 33 (1986) 221.

[6] G. Kido, R. Tanaka and Y. Nakagawa, ibid. 385.

[7] G. Kido, Y. Nakagawa, Proc. Inter. Conf. Magnet Technology (Zurich, 1985) p. 821.

[8] Y. Oeda, M. Takeda, N. Kuroda, G. Kido, Y. Nishina and Y. Nakagawa, this issue, $20 \mathrm{~B}-02$.

[9] R. L. Greene, D. D. Shell, W. M. Yen, A. L. Schawawlow and R. M. White, J. Appl. Phys. $37(1966) 1229$.

[10] I. Mogi, N. Kojima, T. Ban, I. Tsujikawa, G. Kido, M. Takeda and Y. Nakagawa, this issue, 20B-23. 